had isolated microvascular angina, 25 (17\%) had isolated vasospastic angina, $31(20 \%)$ had both (MVA \& VSA) only 17 (11\%) had non-cardiac chest pain. Myocardial bridging of coronary artery was found in $22(15 \%)$. Multivariate predictors of MVA included typical angina, inducible ischaemia but traditional cardiovascular risk factors were not associated. Smoking and age were independent predictors of VSA.

Conclusion The majority of patients with symptoms and/or signs of ischemia and no obstructive disease have a diagnosis of microvascular and/or vasospastic angina. Traditional cardiovascular risk scores have limited discrimination for disorders of coronary vasomotion.

Conflict of Interest Nil

\section{ANIMATION-SUPPORTED CONSENT IN PATIENTS UNDERGOING CORONARY ANGIOGRAPHY AND ANGIOPLASTY}

${ }^{1}$ Prerna Singh*, ${ }^{2}$ David Wald, ${ }^{3}$ Vanessa Cobb. ${ }^{1}$ Barts Health NHS Trust; ${ }^{2}$ Barts Health NHS Trust, ${ }^{3}$ Barts Health NHS Trust

\subsection{6/heartjnl-2019-BCS.49}

Introduction Patient understanding of angiography and angioplasty is often incomplete at the time of consent. Language barriers and time constraints are significant obstacles. New approaches are needed to support communication and decision making. To this end, we developed a multi-language animation describing the benefits, risks and alternatives of the procedures (please click to view www.explainmyprocedure.com). We introduced the animation into practice in a pilot quality improvement project and assessed patients' understanding of the procedure before and after its introduction.

Methods Forty consecutive patients were interviewed, twenty who had watched the animation prior to their procedure and twenty who had not, to determine whether they felt completely informed, understood the benefits, the risks and the alternative treatments. Responses to these four questions were analysed categorically and ratios calculated for the animation group compared with the non-animation group (with 95\% confidence intervals). STATA V10 was used for all analyses.

Results Table 1 shows that patients in the animation group (mean age 64 years) and in the no animation group (mean age 68 years) were well matched. Figure 1 shows that understanding of the procedure was greater in the animation group across all four questions. In the animation group there was a statistically significant 3 -fold greater understanding of the risks

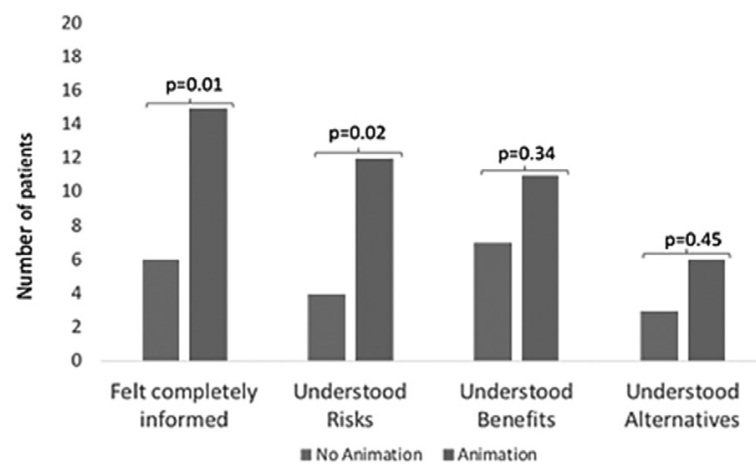

Abstract 51 Figure 1 Patient understanding of the procedure in the animation and no animation groups
Abstract 51 Table 1 Clinical characteristics of the forty patients according to whether they were or were not shown the animation before their procedure

\begin{tabular}{|l|c|c|}
\hline Male & $\begin{array}{c}\text { Not shown Animation } \\
(\mathbf{n = 2 0 )}\end{array}$ & $\begin{array}{c}\text { Shown Animation } \\
(\mathbf{n = 2 0})\end{array}$ \\
\hline Female & 17 & 13 \\
\hline Angiogram & 3 & 7 \\
\hline $\mathrm{PCl}$ & 15 & 13 \\
\hline Elective & 5 & 7 \\
\hline Previous Angiogram & 20 & 19 \\
\hline English Speaker & 7 & 8 \\
\hline
\end{tabular}

$p$ value not significant for any differences in variables between two groups

of the procedure (ratio 3.0 (95\% CI 1.2 to 7.7 ), $\mathrm{p}=0.023$ ) and a 2.5 -fold increase in the proportion of patients who felt completely informed before giving consent (2.5 (1.2 to 5.1), $\mathrm{p}=0.01)$. There were directional, but non-significant, increases in understanding of the benefits and alternatives of the procedure; ratios 1.6 (0.8 to 3.2 ), $\mathrm{p}=0.34$ and 2.0 (0.6 to 6.9), $\mathrm{p}=0.45$, respectively.

Conclusion Viewing animations of angiography and angioplasty before consent was associated with a greater understanding of the procedures and the associated risks. Multi-language narration has the potential to further improve communication surrounding consent. The approach is not limited to cardiology and has the potential to be applied to all specialties in medicine.

Conflict of Interest No conflict of interest

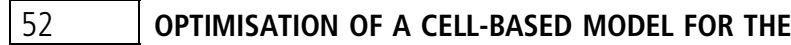 CHARACTERISATION OF NOVEL DRUG-FREE STENT COATINGS}

${ }^{1}$ Daniel Longhorn*, ${ }^{2}$ Susan Currie, ${ }^{3}$ Christopher McCormick. ${ }^{1}$ University of Strathclyde; ${ }^{2}$ University of Strathclyde; ${ }^{3}$ University of Strathclyde

\subsection{6/heartjnl-2019-BCS.50}

Current pharmacological approaches aimed at improving stent designs are limited by their inability to reduce endothelial damage following stent placement and improve healing. Here, we consider in vitro approaches that could be used to assess the effects of novel anti-oxidant stent coatings on damaged endothelial cells following stent placement. Oxidative stress is a physiological response to inflammation and can result in activation of intracellular signalling molecule, leading to pathological effects. One such molecule is calcium/calmodulin dependent protein kinase II $\delta$ (CaMKIII). Hyper-activation of CaMKII is known to be directly linked to disease progression in the heart and it has been implicated in endothelial dysfunction. It is therefore of interest to examine how novel drugfree stent coatings might reduce or reverse oxidative damage following stent placement and whether CaMKII may be a potential target. Our initial experiments have used human umbilical vein endothelial cells (HUVECs), challenged with inflammatory and oxidative stress as a model for future investigations with novel stent coatings.

Novel stent-coatings were generated on $200 \mu \mathrm{m}$ stainless steel wires $(316 \mathrm{~L}$ medical grade), and their antioxidant activity assessed at $8 \mathrm{~h}$ and $24 \mathrm{~h}$ using a DPPH (2,2-diphenyl-1-picrylhydrazyl) colorimetric assay and expressed as a percentage of scavenged to total radicals. Antioxidant activity was observed 
from the novel coatings, with $45 \pm 1.8 \%$ and $87 \pm 3.2 \%$ of radicals scavenged at 8 and $24 \mathrm{~h}$ respectively (one way ANOVA, $n=3, p<0.05)$. Antioxidant activity was sustained over 5 days. HUVECs were cultured onto coated stainless steel strips and imaged using immunofluorescence to assess their attachment and viability.

To model pro-inflammatory and pro-oxidative stress conditions, HUVECs were treated with IL-1 $\beta$; which is known to be elevated in both conditions. ROS generation in HUVECs in response to IL-1 $(3-10 \mathrm{ng} / \mathrm{ml} ; 1-6 \mathrm{~h})$ stimulation was assessed using a DCFDA (2',7'-dichlorofluorescin diacetate)based assay kit (Abcam, UK). IL-1 $\beta$ stimulated ROS production in a time and dose dependant manner with optimal effects at $3 \mathrm{ng} / \mathrm{ml}$ after 6 hours $(3.3 \pm 0.73$-fold increase between stimulated and unstimulated cells, one way ANOVA, $\mathrm{n}=3, \mathrm{p}<0.05)$. Parallel assessment using quantitative immunoblotting of CaMKII $\delta$ in HUVECs showed the enzyme was expressed and that activation (via phosphorylation or oxidation) can be detected. Increased phosphorylation of P65 in response to IL-1 $(10 \mathrm{ng} / \mathrm{ml} ; 15 \mathrm{~min}-6 \mathrm{~h})$ stimulation was also detected, indicating increased pro-inflammatory signalling.

In conclusion, we have established an effective cell-based model for mimicking the oxidative stress that can occur following stent placement and identified CaMKII $\delta$ as a target protein. This approach will be applied to other more physiologically relevant cell types and may be used to examine the desired therapeutic effects of novel stent coatings in vitro. Conflict of Interest N/A

\section{VIRTUAL (COMPUTED) FFR AND VIRTUAL CORONARY INTERVENTION (VCI) VS ANGIOGRAPHY FOR GUIDING PCI: A VIRTUAL STUDY}

${ }^{1}$ Rebecca Gosling*, ${ }^{2}$ Paul Morris, 'Patricia Lawford, ${ }^{1}$ Rodney Hose, ${ }^{1} J u l i a n$ Gunn. ${ }^{1}$ University of Sheffield; ${ }^{2}$ Department of Infection, Immunity and Cardiovascular Disease, Univeristy of Sheffield

\subsection{6/heartjnl-2019-BCS.51}

Introduction Using fractional flow reserve (FFR) to guide percutaneous coronary intervention (PCI) improves outcomes and reduces costs. In FAME, FFR guidance reduced the total length of stent per patient from $52 \mathrm{~mm}$ to $38 \mathrm{~mm}$ and the number from 2.7 to $1.9(1)$, yet FFR is currently used in $<10 \%$ of cases (2). Angiography-based virtual (v) FFR solutions permit less invasive physiological assessment and lend themselves to virtual coronary intervention (VCI) (Figure 1). VCI has been shown to predict the response to PCI with a high degree of accuracy(3). In this study, we sought to determine the potential impact of vFFR and VCI on real world stenting.

Methods Patients who had undergone PCI without FFR guidance were identified from the Sheffield archive. A 3D reconstruction of the diseased artery was generated from the angiogram. Baseline vFFR was calculated using computational fluid dynamics (CFD) analysis(4). If vFFR was $<0.80$, VCI was performed(3). Three PCI strategies were modelled. First, the actual PCI procedure was replicated. Second, the FFRmax was determined; the minimal amount of stenting to achieve the best possible FFR(5). Third, the optimal strategy was determined; the minimal amount of stenting to achieve a post VCI FFR $>0.90$ (6). For each strategy, the total number and length of stent per patient was determined and compared to the actual procedure, which was conducted in the normal way, guided by the angiogram alone.

Results Forty-three patients (56 vessels) were studied. Mean vFFR pre-PCI was $0.74 \pm 0.16$.

Twenty-four (43\%) vessels had a baseline FFR $>0.80$. For the actual procedure, mean post-PCI vFFR was $0.90 \pm 0.09$. The number of stents per patient was $1.4 \pm 0.6$. Total stent length per patient was $29 \pm 15 \mathrm{~mm}$. Mean FFRmax was $0.92 \pm 0.07$. FFRmax was $0.02 \pm 0.03$ higher than the corresponding measured post-PCI FFR. When the virtual procedure was planned to achieve FFRmax, the number of stents per patient was $0.9 \pm 1.0 \quad(\mathrm{p}=0.003)$. Total stent length per patient was $22 \pm 27 \mathrm{~mm}(p=0.04)$. When the virtual procedure was planned to achieve a post VCI FFR $>0.90$, the number of stents per patient was $0.93 \pm 1.02(\mathrm{p}=0.002)$. Total stent length per patient was $20 \pm 25 \mathrm{~mm}(\mathrm{p}=0.01)$.

Conclusion In our cohort, $43 \%$ of vessels had a vFFR $>0.80$ suggesting PCI could have been avoided. Using vFFR and VCI to plan PCI led to a significant reduction in the total number and length of stents recommended per patient. Further work on a larger cohort is required to determine if these findings would translate to improved clinical outcomes.

Figure 1: The 3D reconstruction of the artery is displayed and the operator marks the location where they wish to deploy a stent identified by the red and the blue markers (A). The operator can adjust the radius of the desired virtual stent in the box labelled 'stent size'. The length can be altered by moving the position of the red and blue dots. In the example shown, a $3.0 \mathrm{~mm} \times 20 \mathrm{~mm}$ virtual stent has been inserted. The virtually stented artery is shown overlaying the original vessel in panel B. Reproduced from JACC: Cardiovascular Imaging (3) under creative commons license CC BY 4.0

Conflict of Interest None

\section{Abstract 53 Figure 1}

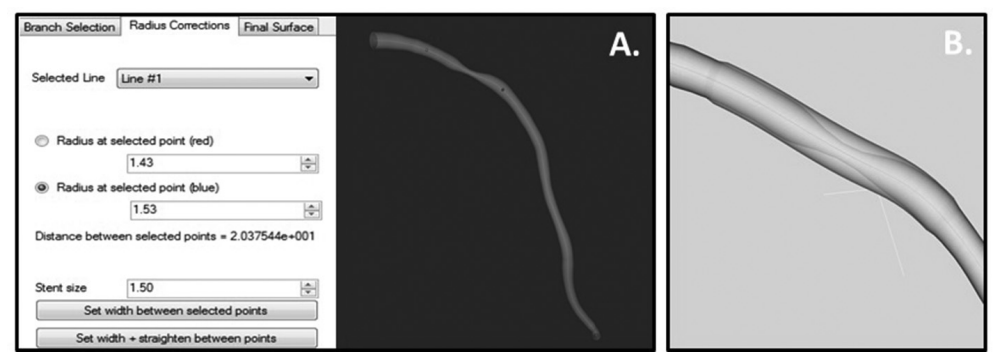

